\title{
Late complete atrioventricular block after hybrid perimembranous ventricular septal defect closure in a neonate
}

\author{
Michał Gałeczka ${ }^{1}$, Mateusz Knop ${ }^{1}$, Roland Fiszer ${ }^{1}$, Alina Zdrzałek-Skiba ${ }^{1}$, \\ Szymon Pawlak ${ }^{2}$, Jacek Białkowski ${ }^{1}$
}

'Department of Congenital Heart Defects and Paediatric Cardiology, SMDZ in Zabrze, Medical University of Silesia, Silesian Centre for Heart Diseases, Zabrze, Poland

2Department of Cardiac, Vascular and Endovascular Surgery and Transplantology, SMDZ in Zabrze, Medical University of Silesia, Silesian Centre for Heart Diseases, Zabrze, Poland

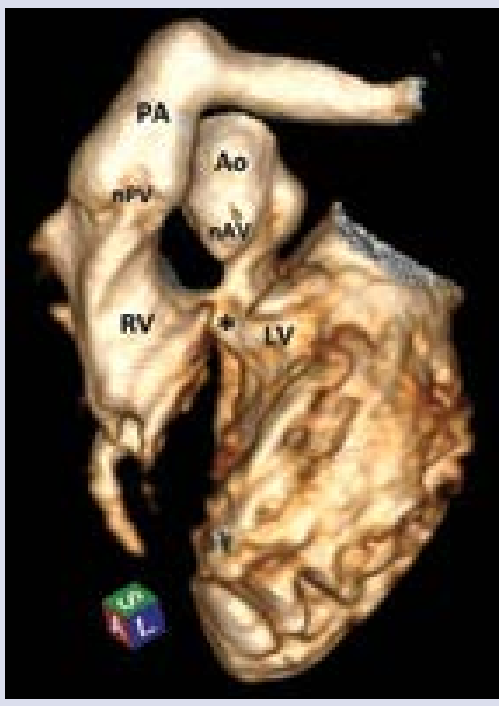

Figure 1. Computed tomography three-dimensional reconstruction. Ventricular septal defect — asterisk; Ao - aorta; LV - left ventricle; nAV neoaortic valve; $\mathrm{nPV}$ - neopulmonary valve; PA — pulmonary artery; RV right ventricle

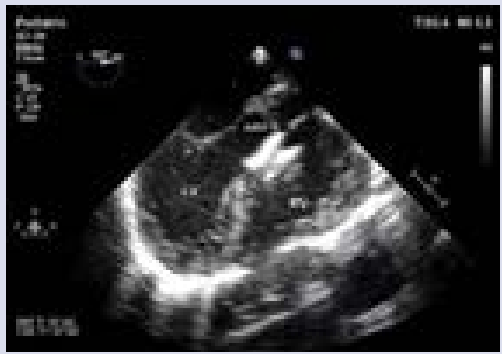

Figure 2. Transoesophageal echocardiography; 7-mm Hyperion ${ }^{\mathrm{TM}}$ VSDO Perimembranous Occluder released in the ventricular septal defect; nAV neoaortic valve; LV - left ventricle; $\mathrm{RV}$ - right ventricle
We present a case of a 3.5-kg male newborn with transposition of the great arteries and 6-mm subpulmonary ventricular septal defect (VSD). On the ninth day after birth he had arterial switch surgery. However, VSD correction was unsuccessful (lack of proper visualisation of the defect and difficult access). In the postsurgical period heart failure worsening was observed due to significant left-to-right shunt. In order to delineate the exact anatomy of the defect, computed tomography was performed (Fig. 1). The heart team decided to close the defect in a hybrid approach. In the third month of life the $4.5-\mathrm{kg}$ patient was readmitted. After sternotomy the right ventricular outflow tract was punctured on the beating heart under transoesophageal echocardiography guidance, which showed a 7 -mm VSD located within $5 \mathrm{~mm}$ of the neoaortic valve (Suppl. Video 1 - see journal website). The defect was crossed with a J-tip guidewire and, subsequently, an 8-F delivery sheath. Then, a 7-mm Hyperion ${ }^{\text {tm }}$ VSDO Perimembranous Occluder was deployed (Comed B.V., Netherlands/Lepu MT Company, China; size of symmetrical discs $-11 \mathrm{~mm}$, length of connecting waist $-3.5 \mathrm{~mm}$ ). Meanwhile, a short episode of two-to-one atrioventricular block (AVB) occurred, but the sinus rhythm recovered quickly. No neoaortic valve regurgitation occurred, but an insignificant residual leak was observed (Suppl. Video $2-$ see journal website). Taking into consideration previous difficulties with the VSD closure, the decision to release the implant was made (Fig. 2). Clinical improvement and no conduction disturbances in multiple 24-h Holter electrocardiography (ECG) recordings were seen in the periprocedural period and in four-month follow-up. However, five months after the procedure the 6.3-kg, eight-month-old asymptomatic patient was readmitted with complete AVB and trivial residual shunt recognised during routine check-up (Suppl. Video 3 - see journal website). A 24-h Holter ECG revealed mean rhythm of $54 \mathrm{bpm}(45-70 \mathrm{bpm})$ with two-to-one conduction and intermittent complete AVB. The patient needed an urgent implantation of an epicardial permanent pacemaker (Fig. 3). Perimembranous VSD is the most common congenital heart disease. Hybrid VSD closure is a feasible and safe approach with less haemodynamic compromise in comparison to surgery. It enables the application of occluders in small children who cannot accommodate the large vascular loading sheaths needed in transcatheter approach [1]. Complete AVB is a well-recognised and most frequent major adverse event after VSD closure with occluders, affecting $3.3 \%$ to $4.5 \%$ of patients [1-3]. The direct pathomechanism of this phenomenon has not been fully established. Mechanical compression in the early postprocedural period and an inflammatory response with extensive scar formation resulting in conduction bundle damage are suspected [2]. Steroid therapy has a proven efficacy in immediate onset of complete AVB. Interestingly, the return of sinus rhythm after such treatment was reported in a patient in whom delayed complete AVB occurred seven months post procedure [1]. We believe that any atrioventricular conduction disturbances during perimembranous

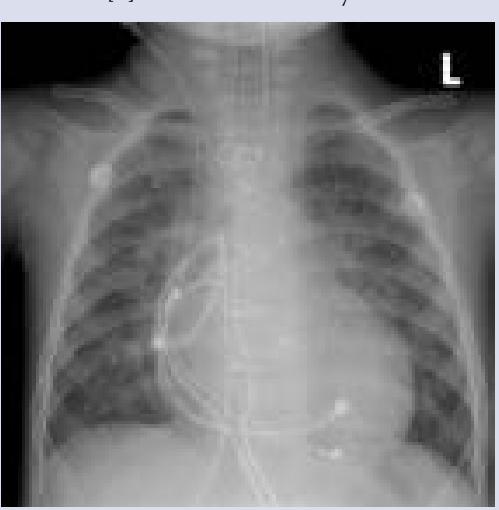

Figure 3. Chest $\mathrm{X}$-ray after epicardial pacemaker implantation or muscular VSD closure in a hybrid or transcatheter approach may lead to AVB progression in the follow-up.

\section{References}

1. Tao K, Lin Ke, Shi Y, et al. Perventricular device closure of perimembranous ventricular septal defects in 61 young children: early and midterm follow-up results. J Thorac Cardiovasc Surg. 2010; 140(4): 864-870, doi: 10.1016/j. jtcvs.2010.05.013, indexed in Pubmed: 20561638

2. Walsh MA, Bialkowski J, Szkutnik M, et al. Atrioventricular block after transcatheter closure of perimembranous ventricular septal defects. Heart. 2006; 92(9): 1295-1297, doi: 10.1136/hrt.2005.084988, indexed in Pubmed: 16449504.

3. Butera G, Carminati M, Chessa M, et al. Percutaneous closure of ventricular septal defects in children aged $<12$ : early and mid-term results. Eur Heart J. 2006; 27(23): 2889-2895, doi: 10.1093/eurhearti/ehl340, indexed in Pubmed: 17053007.

\section{Address for correspondence:}

Michał Gałeczka, MD, Department of Congenital Heart Defects and Paediatric Cardiology, Silesian Centre for Heart Diseases, ul. M. Curie-Skłodowskiej 9 , 41-800 Zabrze, Poland, tel: +48 609345 681, e-mail: michalgaleczka@gmail.com

Conflict of interest: none declared

Kardiologia Polska Copyright (c) Polish Cardiac Society 2018 